\title{
Ultrafast Relaxation Dynamics of the Ethylene Cation $\mathrm{C}_{2} \mathrm{H}_{4}^{+}$- Supporting Information
}

\author{
André Ludwig, ${ }^{\dagger}$ Elisa Liberatore,,$^{\ddagger}$ Jens Herrmann, ${ }^{\dagger}$ Lamia Kasmi $^{\dagger}$ \\ Pablo López-Tarifa, ${ }^{\ddagger}$ Lukas Gallmann, ${ }^{\dagger}{ }^{\top}$ Ursula Rothlisberger ${ }^{\ddagger}$ Ursula Keller, $^{\dagger}$ \\ and Matteo Lucchini*, $\dagger$ \\ $\dagger$ Department of Physics, ETH Zurich, 8093 Zurich, Switzerland \\ $\ddagger$ Laboratory of Computational Chemistry and Biochemistry, EPFL, 1015 Lausanne, \\ Switzerland \\ IInstitute of Applied Physics, University of Bern, 3012 Bern, Switzerland \\ E-mail: mlucchini@phys.ethz.ch
}




\section{Experimental Methods}

The experimental setup ${ }^{1}$ is based on a Ti:sapphire chirped-pulse amplification laser system that delivers 22 -fs IR pulses, centered at a wavelength of $786 \mathrm{~nm}$ with pulse energies of up to $1.35 \mathrm{~mJ}$ and a repetition rate of $1 \mathrm{kHz}$. We split up the incoming beam and use the majority of the intensity for high-order harmonic generation in a gas cell filled with about 15 mbar of xenon which leads to the emission of a harmonic spectrum with photon energies between 20 and $30 \mathrm{eV}$. A 200-nm thick tin (Sn) filter is optionally used to further reduce the spectral range of the excitation pulse to photon energies below $24.5 \mathrm{eV}$. The weaker part of the IR beam travels through a delay line that is controlled by a piezo-driven retro reflector in a range equivalent to $210 \mathrm{fs}$, with a resolution down to $0.02 \mathrm{fs}$. The IR and XUV beams are recombined through a drilled mirror and then focused by a toroidal mirror into the target gas under investigation. We use a mixture of $9 \% \mathrm{C}_{2} \mathrm{H}_{4}$ and $91 \%$ of helium (He), with the latter being used as buffer gas in order to prevent cluster formation and achieve an independent signal for the time-zero calibration. The time-zero calibration is based on the oscillating $\mathrm{He}^{+}$yield around the overlap of the XUV and IR pulses by means of a time-frequency analysis and a fit to the maximum of the 4-IR-photon contribution. ${ }^{2}$ This method enables a time-zero accuracy below $1 \mathrm{fs}$. We collect the positively charged ion fragments by means of time-of-flight mass spectrometry. The use of a tuning-fork chopper in the IR arm allows us to acquire XUV only reference ion yields in alternation with the XUV-pump-IR-probe data which significantly increases the signal-to-noise ratio of the relative ion yields. We show the relative ion yield as function of the pump-probe delay, which is equivalent to the IR-induced change of the ion signal relative to the XUV-only reference: $S_{\text {rel }}=\left(S_{\mathrm{XUV}+\mathrm{IR}}-S_{\mathrm{XUV}}\right) / S_{\mathrm{XUV}}$.

\section{Cross-correlation of pump and probe pulses}

We perform a cross-correlation by performing a RABBITT experiment. ${ }^{3,4}$ Ar atoms are ionized by the attosecond pulse train in the presence of the delayed IR pulse. The results 


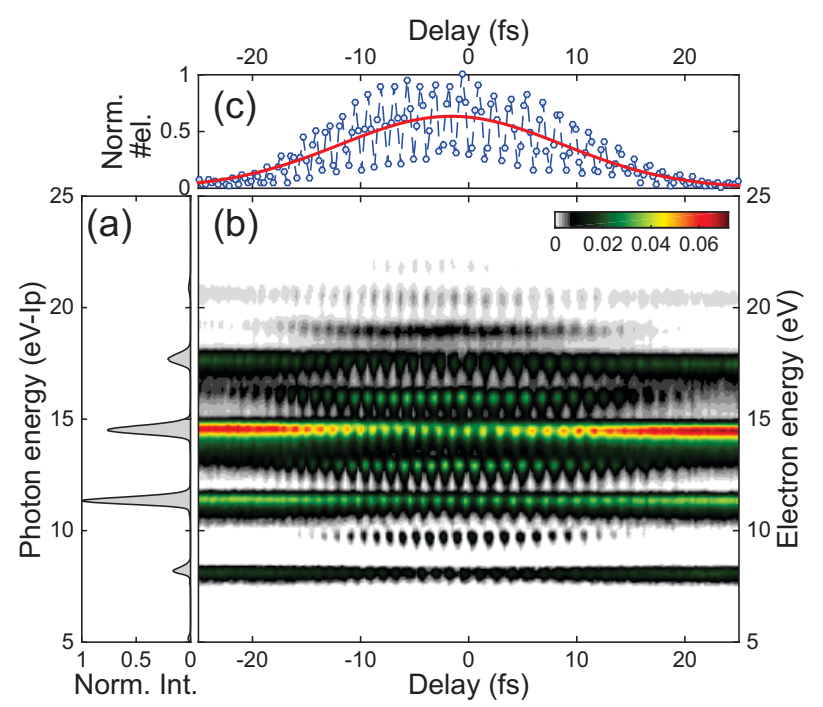

Figure 1: Cross-correlation measurement (RABBITT) obtained by ionizing Ar atoms. (a) Harmonic spectrum. (b) Photoelectron yield as a function of the delay with a 22-fs IR pulse. As one can notice, for small delays an oscillating signal (sideband) appears in between the main peaks corresponding to the direct ionization by the harmonics and oscillate with a period of $\sim 1.25 \mathrm{fs}$. (c) Energy integrated sideband signal centered around $16 \mathrm{eV}$ (blue curve) together with a Gaussian fit (red curve) giving a FWHM of $\sim 24.5 \pm 2 \mathrm{fs}$.

are reported in Fig. 1. For big absolute values of the pump-probe delay, discrete peaks corresponding to direct ionization via absorption of one XUV photon characterize the electron spectra. These peaks lie at the energy position of the odd multiples of the IR fundamental frequency minus the ionization potential of Ar (compare the harmonic spectrum in Fig. 1a) with Fig. 1b)) and are therefore spaced by two IR photons. For small values of the pumpprobe delay a sideband signal appears between the direct ionization peaks corresponding to the positions of the even multiples of the fundamental IR frequency. For a given sideband order, there are two distinct paths that lead to the same final state: absorption of the lower harmonic plus absorption of one IR photon or absorption of the upper harmonic plus emission of one IR photon. Due to the coherence of the process the two paths interfere giving a $2 \omega_{I R^{-}}$ oscillation (see the blue curve in Fig. 1c)). A value for the cross-correlation width can be obtained by fitting the sideband signal with a Gaussian bell (red curve in Fig. 1c)) and gives a full-width-half-maximum (FWHM) of $24.5 \pm 2$ fs. Assuming Gaussian envelopes for both pulses and that the time duration of the APT is half the one of the IR pulse, we extract a 
time duration for the IR beam of $22 \pm 2 \mathrm{fs}$ and of $11 \pm 2 \mathrm{fs}$ for the APT. The retrieved IR time duration is consistent with what obtained with an independent SPIDER measurement. ${ }^{5}$

\section{Experimental Delay Calibration}

The calibration of the time-zero $\left(t_{0}\right)$, when the pump and the probe pulse overlap in time, is of crucial importance for a precise measurement of dynamics on the few-femtosecond timescale, but is not directly accessible from the experiment. Common approaches to calibrate $t_{0}$ rely on additional measurements or the identification of transient features in the data itself. This latter approach was applied in previous experiments leading to a precision of tens of femtoseconds. ${ }^{6}$ An increased time-resolution, as is the case in the presented experiment, requires a more precise determination of the time-zero. We therefore apply a technique that was recently proposed by Herrmann et al., ${ }^{2}$ which is based on multiphoton effects visible in the $\mathrm{He}^{+}$yield from the He buffer gas. Around time-zero, two-color XUV-IR ionization of He is characterized by a yield that oscillates as a function of pump-probe delay. It could

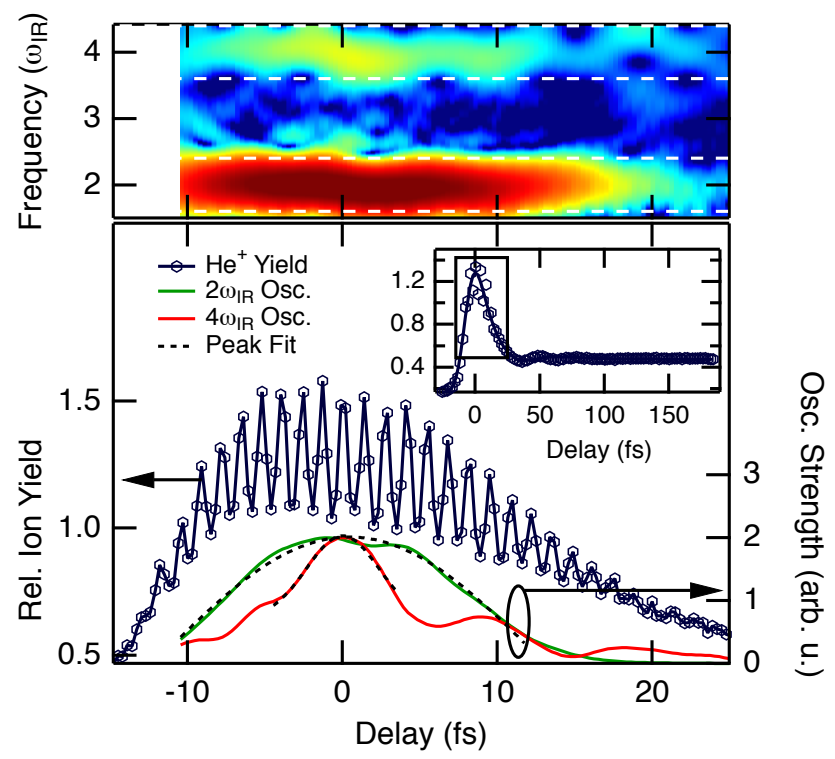

Figure 2: Lower panel: The oscillations in the $\mathrm{He}^{+}$yield visible in a fine scan around temporal pump and probe overlap can be used to precisely calibrate the delay-zero. A time-frequency analysis of the oscillating yield reveals the confined $4 \omega_{\text {IR }}$ contribution that can be fitted by a Gaussian function in order to calibrate the delay axis in the experiment. 
be shown that a time-frequency (Gabor) analysis of these oscillations yields very accurate signatures of the time-overlap with a sub-femtosecond precision. We first use a fine scan with a step width of $0.1 \mathrm{fs}$ around the time-zero to resolve these fast oscillations in the $\mathrm{He}^{+}$ ion yield. A time-frequency analysis (Fig. 2 upper panel) using a sliding-window Fourier transform reveals the delay at which the different frequency components are located. It can be seen, that the dominant contribution stems from the $2 \omega_{\mathrm{IR}}$-oscillation (with $\omega_{\mathrm{IR}}$ being the IR angular frequency), which is accompanied by $4 \omega_{\text {IR }}$-oscillations in a more limited region and is more sensitive to the actual position of the pump-probe overlap. In order to precisely calibrate the time-zero we use a Gaussian fit to the $4 \omega_{\text {IR }}$ oscillation strength whose peak serves as point zero for the following long range fragmentation dynamics scans (Fig. 2 lower panel).

\section{Experimental Results for Different IR Intensities}

In order to characterize the influence of the exact pump and probe parameters, we performed identical scans for decreasing IR intensities between $1.9 \times 10^{12} \mathrm{~W} / \mathrm{cm}^{2}$ and $0.6 \times 10^{12} \mathrm{~W} / \mathrm{cm}^{2}$ as well as for a filtered XUV excitation spectrum limited below $24.5 \mathrm{eV}$ to rule out contributions from the dication (Fig. 3). In all cases, the main features (maximal yields) and their timing are nicely reproduced. The decrease in IR intensity leads to a reduced amplitude of the observed features, quantified as function of IR intensity in Fig. 4. The linear dependence of the dynamical peak amplitudes on the IR intensity indicates, that the probe process constitutes a one-photon process. For clarity, representative error bars are shown for two species only. For the case of the cropped excitation spectrum, the count rate suffers from the reduced XUV flux, estimated to be below $20 \%$ of the unfiltered spectrum.

\section{Computational Methods}

In the Tully's trajectory surface hopping method (TSH) ${ }^{7,8}$ the evolution of a quantum nuclear wave packet is represented by a swarm of independent classical trajectories, each evolving 

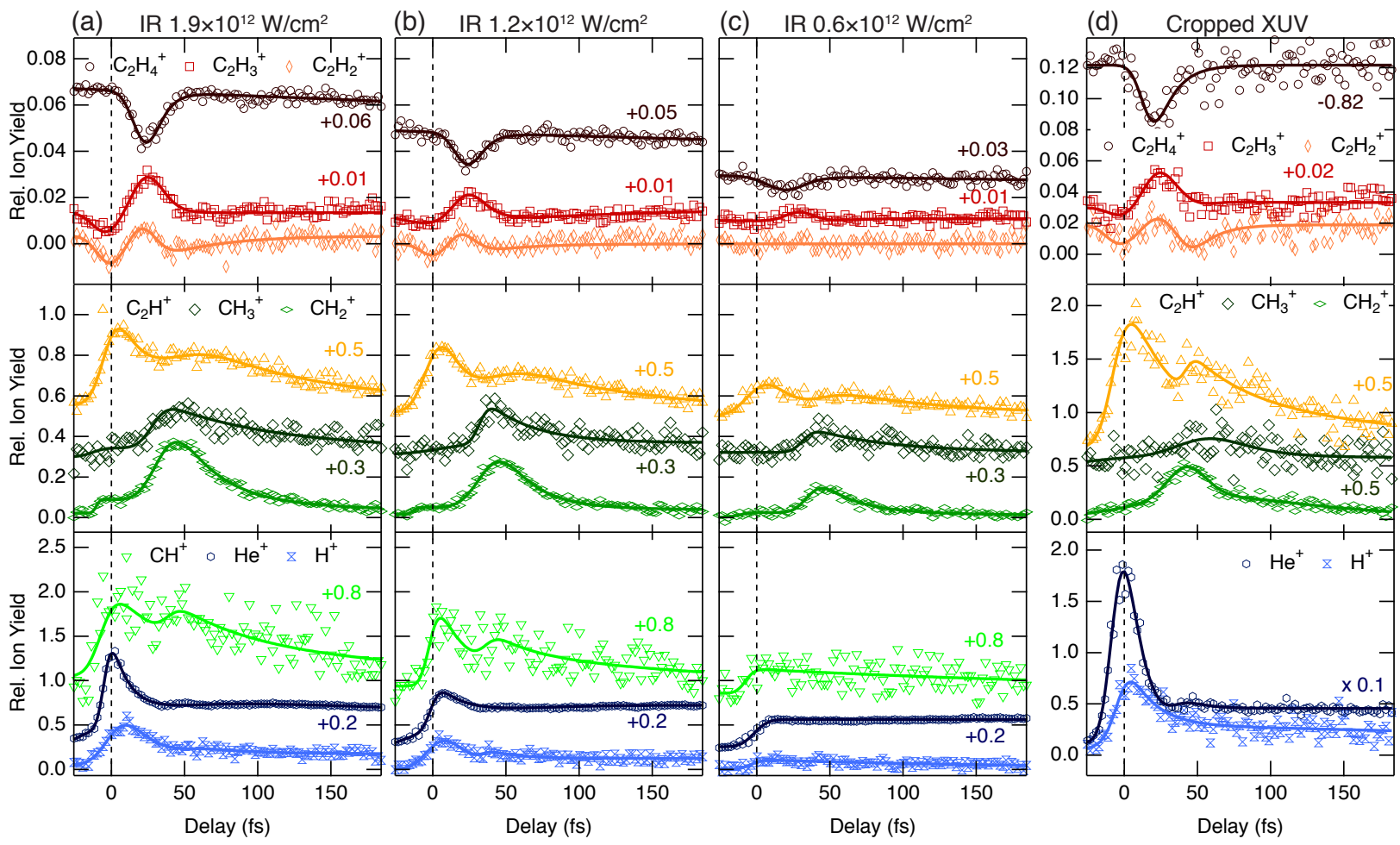

Figure 3: IR-induced change of the ion yields for decreasing IR intensities in the probe pulse (a)-(c) and for the case of the cropped XUV pump spectrum at an intensity of $2.5 \times 10^{12} \mathrm{~W} / \mathrm{cm}^{2}(\mathrm{~d})$. The main features and their time constants are unaffected by the change in intensity and the XUV excitation spectrum.

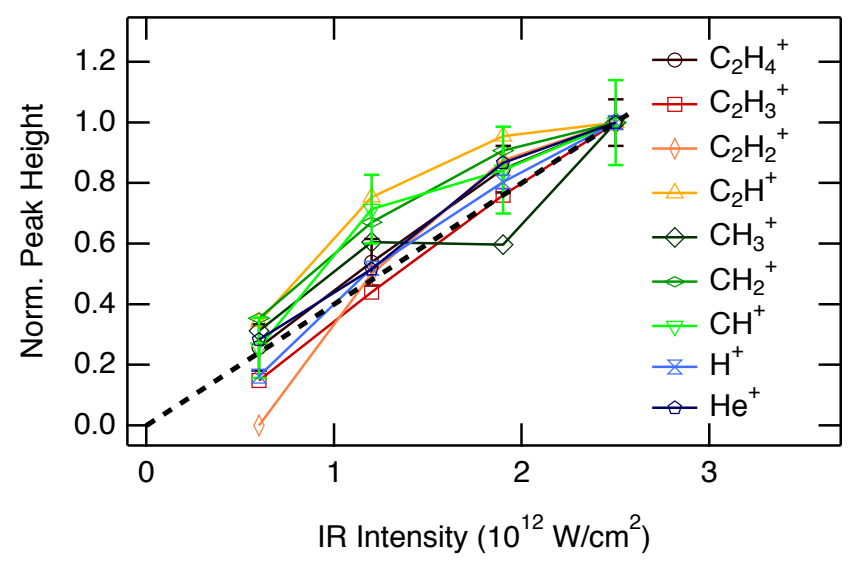

Figure 4: Peak heights of the ion yields representing the signal strength as function of the IR intensity, normalized to their value at the highest intensity. The strength of the signal shows a linear increase with the IR intensities indicating that the probe process represents a single photon process. For clarity, error bars are only shown for the two representative fragments $\mathrm{C}_{2} \mathrm{H}_{4}^{+}$and $\mathrm{CH}^{+}$. 
according to the forces of a specific potential energy surface (PES). Nonadiabatic splitting of the wave packet is mimicked by instantaneous changes ("hops") of the PES on which a given trajectory evolves. Hops between pairs of PES are stochastically decided along the trajectories, with a probability dependent on the strength of the nonadiabatic couplings between the involved PES. Energy conservation after a hop is ensured by velocity rescaling: an attempted hop is rejected if the kinetic energy is insufficient to satisfy this requirement.

Table 1: Comparison of the experimental (first column) and the calculated ionization potentials of ethylene, expressed in $\mathrm{eV}$. Simulation data are obtained with the PBE0 functional and different basis sets: plane waves (PW, box size 20A cutoff 90 a.u.), 6-31G** and 6-311 $+\mathrm{G}^{* *}$. Labels $\tilde{\mathrm{X}}, \tilde{\mathrm{A}}, \tilde{\mathrm{B}}, \tilde{\mathrm{C}}, \tilde{\mathrm{D}}$, and $\tilde{\mathrm{E}}$ refer to the spectroscopic states obtained by removing an electron from one of the valence orbitals.

\begin{tabular}{ccccc}
\hline & Exp. & PW & 6-311++G** & 6-31G** \\
\hline$\tilde{X}$ & 10.68 & 10.52 & 10.51 & 10.45 \\
$\tilde{\mathrm{A}}$ & 12.8 & 12.79 & 12.62 & 12.69 \\
$\tilde{\mathrm{B}}$ & 14.8 & 14.58 & 14.50 & 14.49 \\
$\tilde{\mathrm{C}}$ & 16.0 & 15.95 & 15.84 & 15.89 \\
$\tilde{\mathrm{D}}$ & 19.1 & 19.08 & 18.92 & 18.97 \\
$\tilde{\mathrm{E}}$ & 23.6 & 24.11 & 24.06 & 24.09 \\
\hline
\end{tabular}

In the present work we coupled the TSH method with Linear-Response Time Dependent Density Functional Theory (LR-TDDFT) ${ }^{9}$ for the on-the-fly calculation of electronic energies and nonadiabatic couplings. TDDFT is the time-dependent extension of the Density Functional Theory (DFT) ${ }^{10}$ and as in the ground-state formulation, the accuracy of TDDFT results relies on the level of the exchange correlation functional. After comparison of different hybrid and meta-hybrid functionals, we chose the hybrid PBE0 functional for our calculations. This functional provides vertical ionization potentials for ethylene in very good agreement with the experimental values.

The excitation energies for the cation in the ground state geometries are also well reproduced. In Table 2 the PBE0-TDDFT excitation energies from the ground-state optimized geometries of the cation are compared with the values reported in Joalland et al., ${ }^{13}$ Supporting Material, calculated at a CASSCF and CASPT2 level of theory. For the PBE0 
and the CASSCF data, the deviation from the SA4-CASPT2(11/7) values is shown within parenthesis.

Table 2: Vertical excitations from the ground state of ethylene cation in its optimized geometry. Comparison of different electronic structure methods: PBE0TDDFT (second and third columns, with different basis sets), SAn-CASSCF with different active spaces (fourth and fifth columns) and CASPT2 (sixth column). CASSCF and CASPT2 data from Ref. ${ }^{13}$ Deviations from the CASPT2 values are shown within parenthesis

\begin{tabular}{cccccc}
\hline state & $\begin{array}{c}\text { PBE0 } \\
\left(6-31 \mathrm{G}^{* *}\right)\end{array}$ & $\begin{array}{c}\text { PBE0 } \\
\left(6-311++\mathrm{G}^{* *}\right)\end{array}$ & $\begin{array}{c}\text { SA3- } \\
\text { CASSCF }(11 / 8)\end{array}$ & $\begin{array}{c}\text { SA4- } \\
\text { CASSCF }(11 / 7)\end{array}$ & $\begin{array}{c}\text { SA4- } \\
\text { CASPT2 }(11 / 7)\end{array}$ \\
\hline D1 & $3.06(-0.35)$ & $2.94(-0.47)$ & $3.59(0.18)$ & $3.78(0.37)$ & 3.41 \\
D2 & $4.31(-0.05)$ & $4.30(-0.06)$ & $4.44(0.08)$ & $4.51(0.15)$ & 4.36 \\
D3 & $5.59(0.81)$ & $5.48(0.70)$ & & $5.88(1.10)$ & 4.78 \\
\hline
\end{tabular}

\section{Potential Energy Surfaces Scan}

To further assess the performances of TDDFT on the present system, as well as to obtain a preliminary picture of the possible relaxation pathways, we performed calculations of the four lowest-energy PESs of ethylene cation, namely D0, D1, D2 and D3. For computing and plotting the PESs, we considered the most relevant degrees of freedom in the molecule relaxation and fragmentation processes: the torsional angle $\tau$, the bridging angle $\varphi$, the $\mathrm{C}-\mathrm{H}$ distance, and the $\mathrm{C}-\mathrm{C}$ distance. The molecular geometries are first calculated on a grid with fixed values of two of the variables, via constrained optimization on the ground state D0. All the other degrees of freedom but the two of interest are allowed to relax. The optimization was carried out at a PBE0-DFT level and the grid spacing is $10^{\circ}$ for the angular variables, $0.1 \AA$ for the $\mathrm{C}-\mathrm{H}$ and $\mathrm{C}-\mathrm{C}$ distances. We then computed the excitation energies at the optimized geometries to obtain the D1, D2 and D3 PES. These simulations were performed using the quantum chemistry simulation code GAUSSIAN G09, ${ }^{11}$ using a $6-31 \mathrm{G}^{* *}$ basis set. In agreement with Joalland et al. ${ }^{13}$ the torsional-bridging PES presents two CIs: a planarbridged CI $\left(\tau=0^{\circ}, \varphi=85^{\circ}\right)$ and a twisted CI $\left(\tau=90^{\circ}\right)$. The PES along the C-C and C-H 
distances presents the $\mathrm{CI}$ along the $\mathrm{C}-\mathrm{H}$ coordinate at $1.7 \AA$ and a $\mathrm{C}$ - $\mathrm{C}$ distance of $1.3 \AA$.
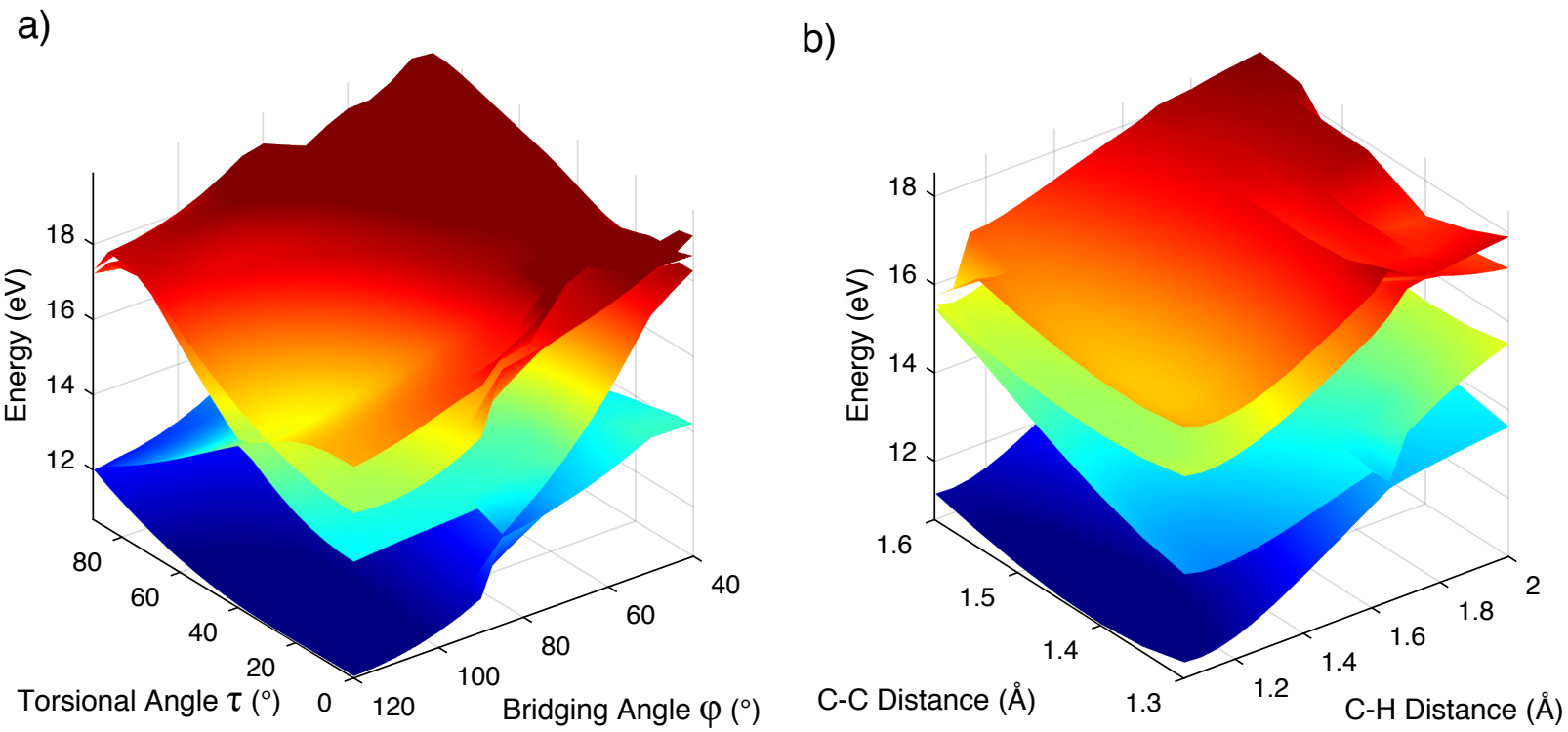

Figure 5: PES scan along the bridging and the torsional coordinates in a) and along the $\mathrm{C}-\mathrm{C}$ and $\mathrm{C}-\mathrm{H}$ bond lengths, respectively. The zero of the energy is the cationic ground state energy, in its optimal geometry.

In Fig. 6 we compare the potential energy scan along the torsional coordinate calculated in present work at PBE0-TDDFT level of theory (left panel) and that of Joalland and coworkers, ${ }^{13}$ obtained at a SA4-CAS(11/7) level of theory (right panel). There is a good agreement on the PES features relevant to the relaxation: the D1/D0 CI at a twist angle of 90 degrees, the D3/D2 and the D2/D1 CIs at around 35 and 45 degrees, slightly shifted towards smaller angles compared to the CASSCF values. The main differences are in the D3 state at torsional angles greater than 50 degrees, were TDDFT energies are smaller than those computed by Joalland et al. and closer to the D2 state. If the torsional coordinate alone is considered, the differences in the PES will not determine a significant deviation in the relaxation dynamics from states D2 and D1. An effect could result on the relaxation from D3, but given the initial angular distributions (close to planar, $\tau=0$ ) and the presence of a D3/D2 CI at smaller angles, we expect it to be minor. 

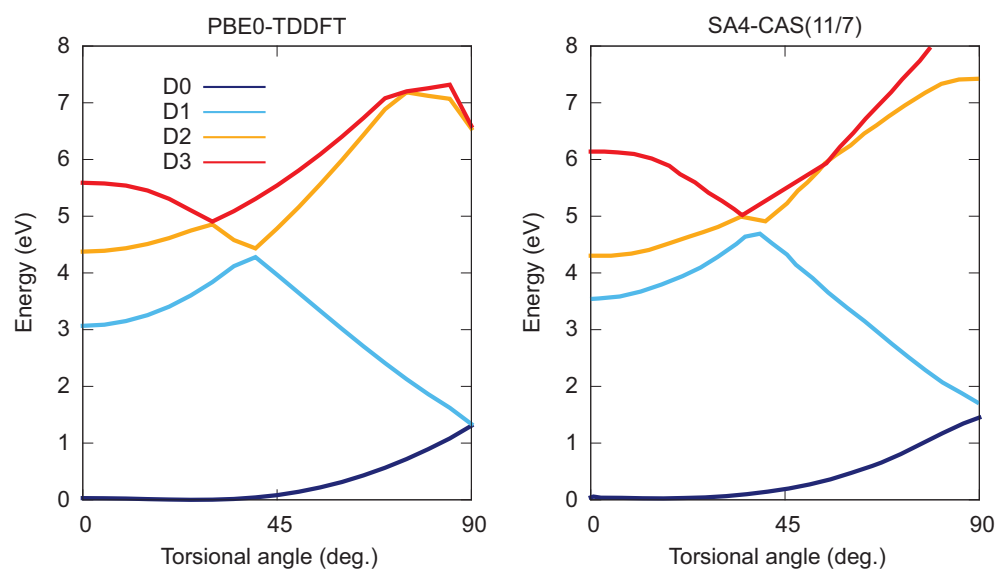

Figure 6: Comparison between the PES of the ground and the first three excited states of ethylene cation along the torsional coordinate, as obtained from PBE0-TDDFT calculations (left panel) and from SA4-CASSCF $(11 / 7)$ in Ref. ${ }^{13}$ (right panel). The geometries are obtained from constrained ground state optimization, in which only the torsional angle is fixed.

\section{TSH simulations}

In TSH calculations, for each one of the excitations relevant for the experiment (D1, D2, D3) we initialized 100 TSH trajectories: starting geometries and velocities were obtained from the Wigner distribution relative to the neutral ethylene in its ground state. Initial geometries are hence of non-equilibrium for the cation, differing also in this from Joalland et al. ${ }^{13}$ The propagation time-step is $0.2 \mathrm{fs}$ and the trajectories were run for a total simulation time of 200 fs. Given the proximity of the PESs at the higher excitations that come into play, we included in the dynamics the calculation of excited states up to D10. Simulations were carried out with the Newton.X package ${ }^{12}$ interfaced with G09 for the calculation of the electronic properties, using a $6-31 \mathrm{G}^{* *}$ basis set. From the TSH trajectories we were able to extract information on the time evolutions of the excited state population, molecular geometries. Furthermore, we analyzed the geometries at the D1/D0 hops, focusing on their characteristics with respect to the maximum $\mathrm{C}-\mathrm{H}$ distance and the torsional angle, as discussed in the paper. Roughly $10 \%$ of the overall hops to the ground state occur when one of the $\mathrm{C}-\mathrm{H}$ bonds is pre-dissociated, suggesting that a CI with this characteristic is reached. An illustration of the molecular conformation when the D1/D0 hop shows an elongated C-H bond is provided 
in Fig. 7-8, referring to trajectories initialized on D2 and D3, respectively. The twisted CI seems instead to play a minimal role only when the molecule is initially excited on D3, as only in some of those trajectories we observe hops occurring in a twisted geometry. These hops are shown in Fig. 9. We observe a few fragmentation events in our dynamics, all occurring in the ground state. H loss do not necessarily involve isomerization or bridging, and only some of the trajectories that have relaxed to D0 through a planar CI (stretched of bridged) are dissociative. The two videos included in the Supporting Information illustrate the nuclear motion along trajectories leading to loss of $\mathrm{H}$ and $\mathrm{H}_{2}$.
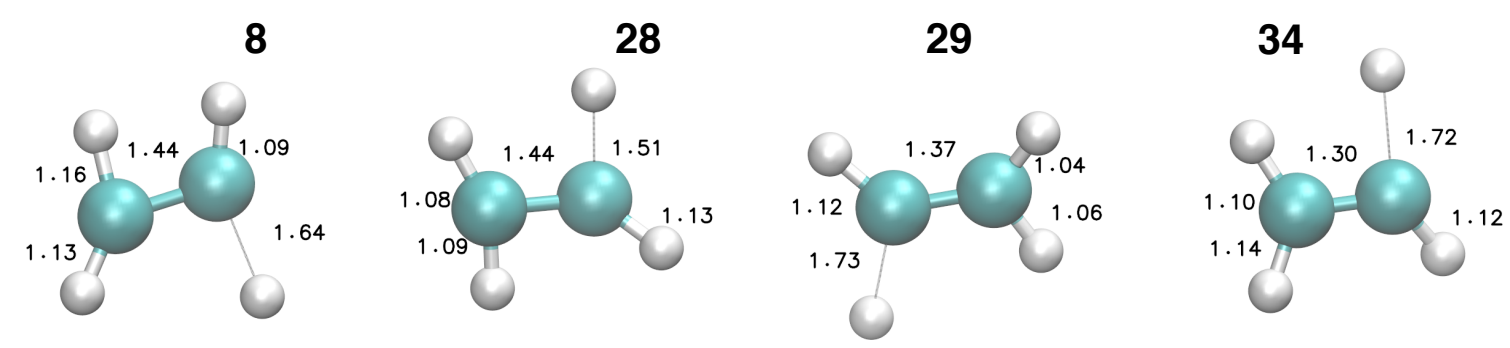

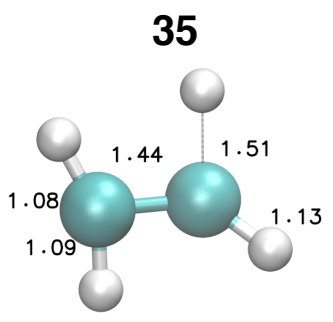

73

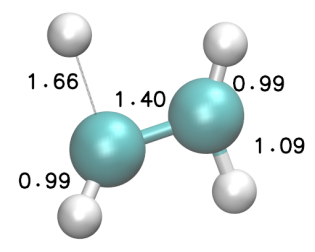

36

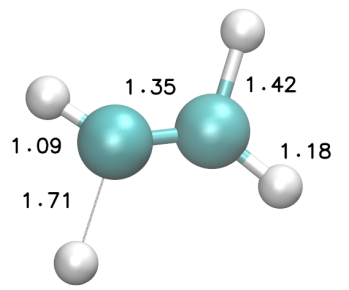

81
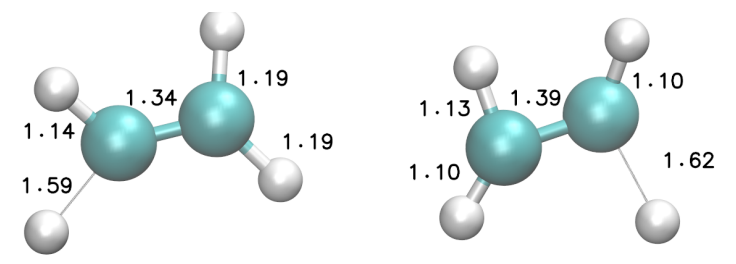

60

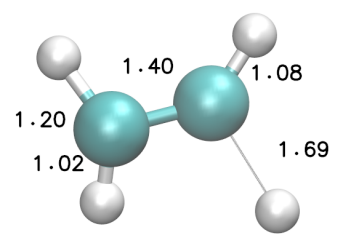

91

94

Figure 7: Ball-and-stick representation of the molecular geometries at the D1/D0 hops that display an over-stretched $\mathrm{C}-\mathrm{H}$ bond, in the trajectories initialized on state D2. Bonds longer than $1.5 \AA$ are represented as dotted lines. The numbers indicate the trajectory identification. The interatomic distances, in $\AA$, are also shown, close to the relative bond. 


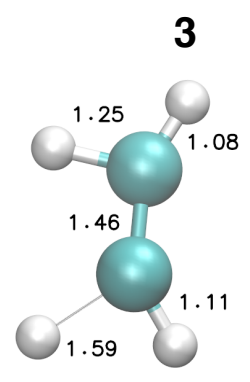

18

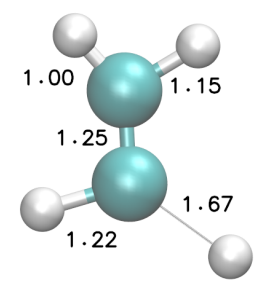

22

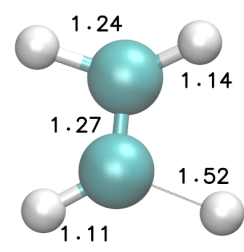

28

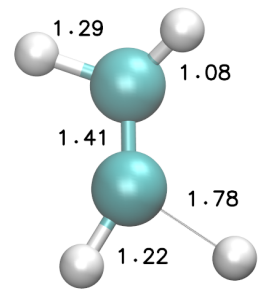

36

38

41

44

79
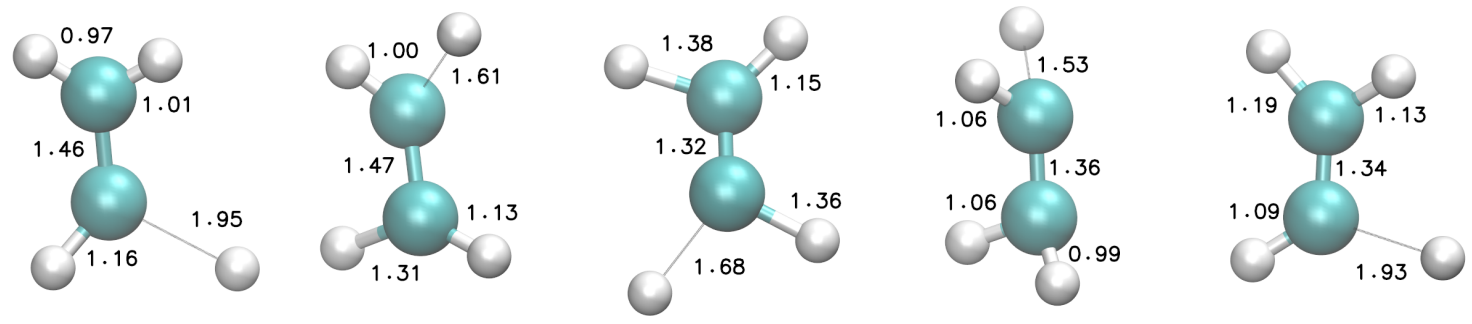

Figure 8: Ball-and-stick representation of the molecular geometries at the D1/D0 hops that display an over-stretched $\mathrm{C}-\mathrm{H}$ bond, in the trajectories initialized on state D3. Bonds longer than $1.5 \AA$ are represented as dotted lines. The numbers indicate the trajectory identification. The interatomic distances, in $\AA$, are also shown, close to the relative bond. 
13

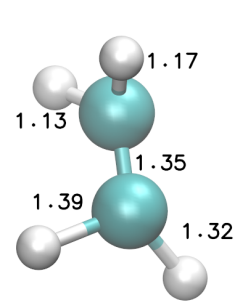

14

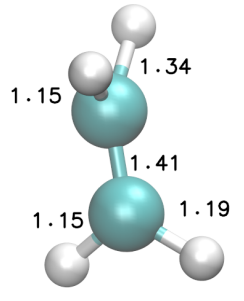

16

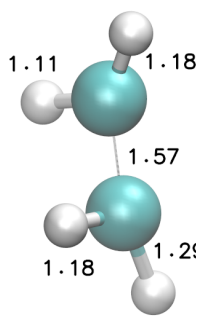

21

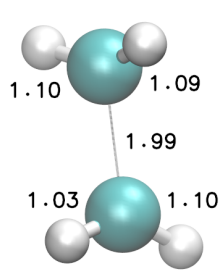

24

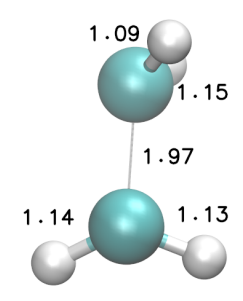

26

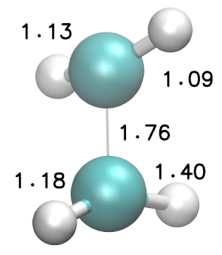

35

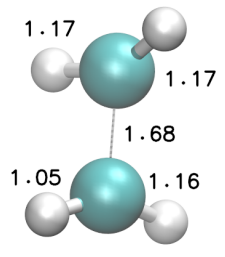

36

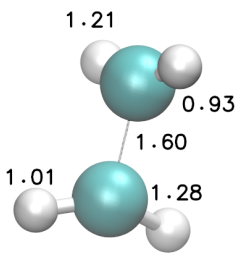

52

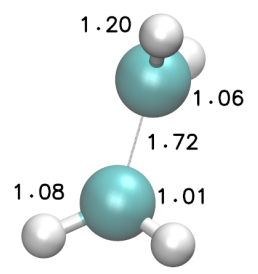

Figure 9: Ball-and-stick representation of the molecular geometries at the D1/D0 hops characterized by a torsional angle close to $90^{\circ}$, in the trajectories initialized on state D3. Bonds longer than $1.5 \AA$ are represented as dotted lines. The numbers indicate the trajectory identification. The interatomic distances, in $\AA$, are also shown, close to the relative bond. 


\section{References}

(1) Locher, R.; Lucchini, M.; Herrmann, J.; Sabbar, M.; Weger, M.; Ludwig, A.; Castiglioni, L.; Greif, M.; Hengsberger, M.; Gallmann, L. et al. Versatile Attosecond Beamline in a Two-foci Configuration for Simultaneous Time-resolved Measurements. Rev. Sci. Instrum. 2014, 85, 013113-1-013113-9.

(2) Herrmann, J.; Lucchini, M.; Chen, S.; Wu, M.; Ludwig, A.; Kasmi, L.; Schafer, K. J.; Gallmann, L.; Gaarde, M. B.; Keller, U. Multiphoton Transitions for Delay-zero Calibration in Attosecond Spectroscopy. New J. Phys. 2015, 17, 013007-1-013007-10.

(3) Paul, P. M.; Toma, E. S.; Breger, P.; Mullot, G.; Auge, F.; Balcou, P.; Muller, H. G.; Agostini, P. Observation of a Train of Attosecond Pulses from High Harmonic Generation. Science 2001, 292, 1689-92.

(4) Muller, H. G. Reconstruction of Attosecond Harmonic Beating by Interference of Twophoton Transitions. Appl. Phys. B: Lasers Opt. 2002, 74, s17-s21.

(5) Iaconis, C.; Walmsley, I. A. Spectral Phase Interferometry for Direct Electric-field Reconstruction of Ultrashort Optical Pulses. Opt. Lett. 1998, 23, 792-794.

(6) Tilborg, J. V.; Allison, T. K.; Wright, T. W.; Hertlein, M. P.; Falcone, R. W.; Liu, Y.; Merdji, H.; Belkacem, A. Femtosecond Isomerization Dynamics in the Ethylene Cation Measured in an EUV-pump NIR-probe Configuration. J. Phys. B: At. Mol. Opt. Phys. 2009, 42, 081002-1-081002-5.

(7) Tully, J. C. Molecular Dynamics with Electronic Transitions. J. Chem. Phys. 1990, 93, 1061-1071.

(8) Barbatti, M. Nonadiabatic Dynamics with Trajectory Surface Hopping Method. Wiley Interdiscip. Rev. Comput. Mol. Sci. 2011, 1, 620-633. 
(9) Runge, E.; Gross, E. K. U. Density-Functional Theory for Time-Dependent Systems. Phys. Rev. Lett. 1984, 52, 997-1000.

(10) Hohenberg, P.; Kohn, W. Inhomogeneous Electron Gas. Phys. Rev. 1964, 136, B864B871.

(11) Frisch, M. J.; Trucks, G. W.; Schlegel, H. B.; Scuseria, G. E.; Robb, M. A.; Cheeseman, J. R.; Scalmani, G.; Barone, V.; Mennucci, B.; Petersson, G. A. et al. Gaussian 09 Revision A.01. Gaussian, Inc.: Wallingford, CT, 2009.

(12) Barbatti, M.; Ruckenbauer, M.; Plasser, F.; Pittner, J.; Granucci, G.; Persico, M.; Lischka, H. Newton-X: a Surface-hopping Program for Nonadiabatic Molecular Dynamics. Wiley Interdiscip. Rev. Comput. Mol. Sci. 2014, 4, 26-33.

(13) Joalland, B.; Mori, T.; Martínez, T. J.; Suits, A. G. Photochemical Dynamics of Ethylene Cation C2H4+. J. Phys. Chem. Lett. 2014, 5, 1467-1471. 